\title{
Haplotype analysis in prenatal diagnosis and carrier identification of Salla disease
}

\author{
Johanna Schleutker, Pertti Sistonen, Pertti Aula
}

\begin{abstract}
Salla disease (SD) is an autosomal recessive disorder in which free sialic acid (N-acetyl neuraminic acid) accumulates in lysosomes. A specific transport mechanism for acidic monosaccharides on the lysosomal membrane has recently been described, but the molecular deficiency causing SD is still unknown. We have previously mapped the SD gene to 6q14-q15 by means of genetic linkage analysis and restricted the positive chromosomal area to less than $100 \mathrm{~kb}$ with linkage disequilibrium mapping. The two best allelic association markers have now retrospectively been used in five prenatal analyses originally studied with sialic acid assays in chorionic villus specimens. In four cases an unaffected fetus was predicted with a probability level of more than $94 \%$, which was in concordance with the biochemical data. One fetus was predicted to be affected with over $96 \%$ probability, as was shown by free sialic acid assays in a CVS sample and in fetal tissues after termination of the pregnancy. Risk calculations incorporating disequilibrium were also used to predict the carrier status in members of six families with previous SD cases, and also in a few cases with no known family history of SD. DNA marker based analysis thus provides a reliable method for risk estimations in prenatal cases and for carrier identification of SD. (f Med Genet 1996;33:36-41)
\end{abstract}

Key words: Salla disease; prenatal diagnosis.

Salla disease (SD), also known as Finnish or adult type free sialic acid storage disorder (MIM 269920), is caused by accumulation of NANA ( $\mathrm{N}$-acetylneuraminic acid) in the lysosomes. ${ }^{1}$ A specific transport mechanism for acidic monosaccharides on the lysosomal membrane has been described and a defect in the putative transport protein of NANA is the likely cause of the disease. ${ }^{23}$ The diagnosis of SD is based on early onset psychomotor retardation associated with ataxia and some other neurological abnormalities. ${ }^{4}$ The progression of the symptoms is relatively slow and SD patients have a near normal life expectancy. ${ }^{56} \mathrm{SD}$ is inherited as an autosomal recessive trait with exceptional enrichment in the Finnish population, particularly in the north-eastern part of the country, the Salla region. A gene frequency of 0.006 has been calculated for the whole of Finland, whereas a considerably higher frequency of 0.013 has been estimated for the high incidence area. ${ }^{7}$ The gene frequencies in other populations are not known, but rare sporadic families have been described. ${ }^{89}$ Lysosomal accumulation of free sialic acid also leads to a phenotypically different clinical entity, the infantile type of free sialic acid storage disease (ISSD). ${ }^{10}$ Prenatal diagnosis of SD and ISSD has so far been based on the increased intracellular free sialic acid content in cultured amniotic fluid cells, ${ }^{11}$ or in CVS samples in ISSD. ${ }^{12-14}$

We have assigned the gene for SD to $6 \mathrm{q} 14-15$ by linkage analyses in Finnish families. ${ }^{715}$ The locus homogeneity of different clinical phenotypes of free sialic acid storage diseases was recently shown in patients from different ethnic backgrounds. ${ }^{16} \mathrm{~A}$ founder effect derived linkage disequilibrium in the Finnish population is evident between the disease locus and the closest markers AFMa336zf9 and AFMb281wg9. Eighty-five percent of Finnish SD patients are homozygous for the 1-3 haplotype of these two microsatellite markers, and there is only one family (out of a total of 50 families) with two affected sisters who lack the 1-3 haplotype and are homozygous for the 1-8 haplotype.

Here we assess the value of linkage disequilibrium in prenatal diagnosis of $\mathrm{SD}$ by calculating retrospectively the risks of being affected in five at risk pregnancies, which were originally studied by free sialic acid assay in uncultured CVS samples. We also applied haplotype analysis to estimate the probabilities of carrier status in family members of SD patients.

In one of the prenatal cases an affected child was predicted, whereas in four instances SD was excluded with probability levels ranging from 96 to $100 \%$. These results correlated well with the original sialic acid data. Carrier identification could be performed in all cases with a high probability level. Haplotype based risk calculation can now be applied to identify carriers of SD particularly in the subpopulation of the high risk area in Finland.

\section{Materials and methods}

PRENATAL DIAGNOSIS FAMILIES

Prenatal diagnosis had been performed in at risk pregnancies by free sialic acid assay on uncultured first trimester CVS samples in four families (fig 1) with an earlier child affected with Salla disease. Sialic acid was assayed with an HPLC method (M Renlund, personal communication). In four pregnancies the sialic acid content was within the control level, the pregnancies went to term, and healthy infants were 

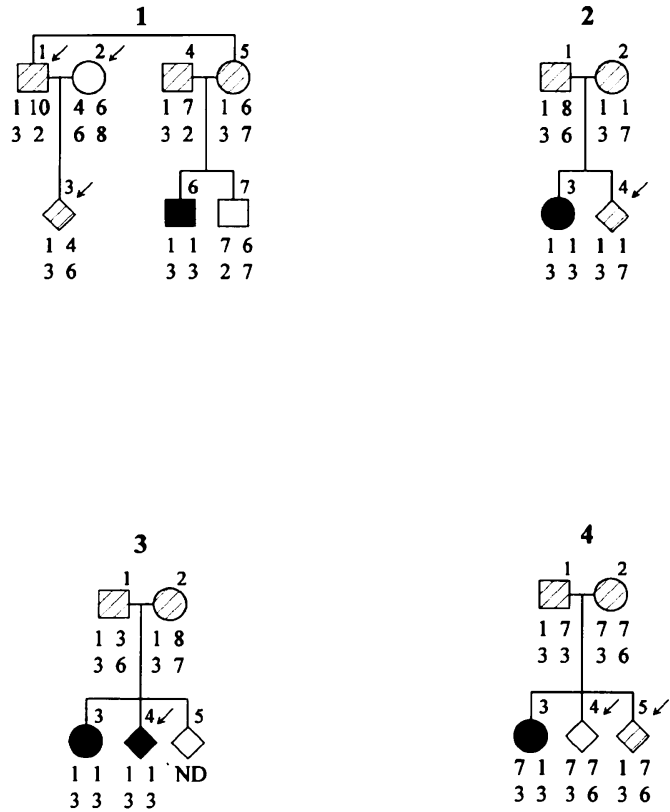

Figure 1 Pedigrees of prenatal diagnosis families. The corresponding haplotypes are given below each subject. Risk was calculated for those subjects marked with an arrow. The blackened symbols indicate a patient with Salla disease, hatched symbols indicate a heterozygote.

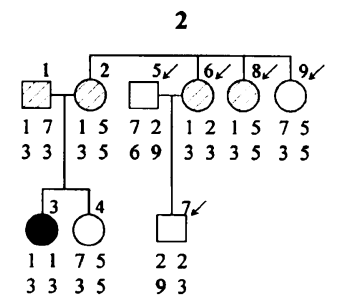

4
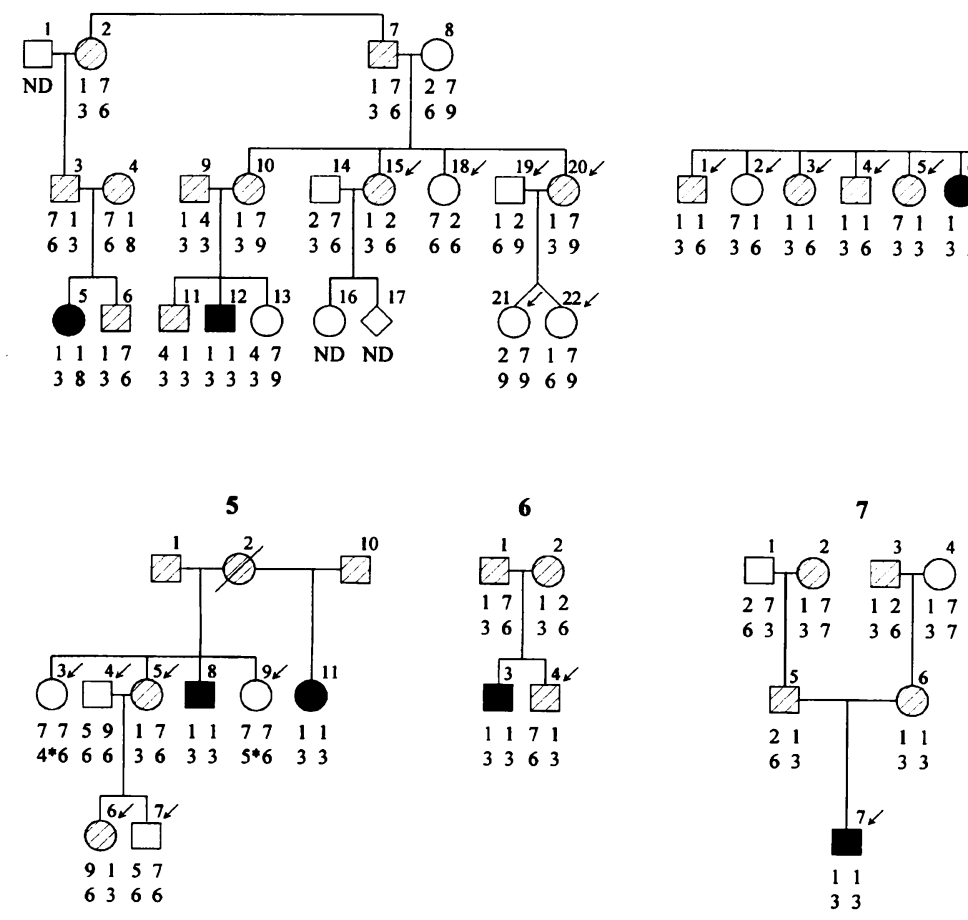

Figure 2 Pedigrees of the families for carrier identification.* denotes a new mutation in one marker allele. Results of family 7 are only presented in the text. delivered. Normal development and the absence of free sialic acid in urine have confirmed the prenatal prediction in these children. In one case (family 3 ) the raised free sialic acid level in a CVS sample indicated that the fetus was affected with SD and the pregnancy was terminated. An increased level of free sialic acid and the presence of enlarged vacuoles in several tissues of the fetus confirmed the diagnosis of SD.

Aliquots of CVS samples were used retrospectively for DNA extraction and haplotype analysis using the two most closely linked markers AFMa336zf9 and AFMb281wg9. DNA samples from family members were studied simultaneously with the fetal samples. Prediction of the fetal status was based on linkage analysis as described below.

\section{CARRIER IDENTIFICATION FAMILIES}

Twenty-two healthy members from five families in which SD segregated requested estimation of the probability of being a carrier of SD. Eight of them were sibs of an SD patient. The pedigrees of the families are shown in fig 2 . In family 2 carrier identification was requested by a 25 year old pregnant woman (6) whose sister had a daughter affected with SD. The husband also originated from the high gene frequency area and, in addition, he may be related to another SD family. Family 3 with second cousins (5 and 12) affected with SD originates from eastern Finland, outside the high risk area. Four family members (indicated in fig 2) requested carrier identification.

In two additional families (6 and 7) haplotype analysis was performed for diagnostic purposes. The newborn baby in family 6 had no signs of SD nor any other abnormalities and the urinary free sialic acid was within normal limits. The parents had refused prenatal studies during the pregnancy. In family 7 the haplotype analysis was performed on a 9 month old male infant whose clinical and sialic acid studies had led to a strong suspicion of SD. He was hypotonic and ataxic. MRI of the central nervous system showed evidence of demyelination and the amount of free sialic acid in urine was above normal limits.

\section{DNA SAMPLES}

DNA was extracted from venous blood in accordance with standard protocols and from second trimester villus biopsy specimens, which were originally taken for the determination of free sialic acid. ${ }^{11}$ In one case the DNA was extracted from a paraffin block using a modification of the method described by Dubeau et al. ${ }^{17}$ Deparaffinisation and rehydration was carried out with $200 \mu$ l xylene, $600 \mu \mathrm{l} 70 \%$ ethanol (twice), followed by short incubation at $55^{\circ} \mathrm{C}$. After incubation a mixture of $75 \mu \mathrm{l}$ $\mathrm{H}_{2} \mathrm{O}, 10 \mu \mathrm{l}$ lysate buffer, $5 \mu \mathrm{l} 10 \%$ Tween, and $10 \mu \mathrm{l}$ proteinase $\mathrm{K}$ was added and the sample was incubated overnight. The enzyme was inactivated at $95^{\circ} \mathrm{C}$ for 10 minutes and 3-5 $\mu \mathrm{l}$ of the solution was used in PCR. 
Table 1 Finnish population frequencies of AFMa336zf9-AFMb281wg9 haplotypes in normal and SD chromosomes adjusted to 0.9870 for normal chromosomes and to 0.0130 for SD chromosomes according to the local SD gene frequency of 0.013

\begin{tabular}{|c|c|c|c|c|c|}
\hline \multicolumn{3}{|c|}{ Normal chromosomes } & \multicolumn{3}{|c|}{$S D$ chromosomes } \\
\hline Haplotype & No & Frequency & Haplotype & No (\%) & Frequency \\
\hline $1-2$ & 2 & 0.020143 & $1-3$ & $66(86 \cdot 0)$ & 0.011143 \\
\hline $1-3$ & 1 & 0.010071 & $1-5$ & $1(1.25)$ & 0.000169 \\
\hline $1-6$ & 2 & 0.020143 & $1-7$ & $1(1 \cdot 25)$ & 0.000169 \\
\hline $1-7$ & 2 & 0.020143 & $1-8$ & $3(3.90)$ & 0.000506 \\
\hline $2-3$ & 3 & 0.030214 & $2-1$ & $1(1 \cdot 25)$ & 0.000169 \\
\hline $2-5$ & 1 & 0.010071 & $2-2$ & $1(1.25)$ & 0.000169 \\
\hline $2-6$ & 10 & $0 \cdot 100714$ & $2-6$ & $1(1 \cdot 25)$ & 0.000169 \\
\hline $2-7$ & 2 & 0.020143 & $5-8$ & $1(1.25)$ & 0.000169 \\
\hline $2-8$ & 1 & 0.010071 & $7-3$ & $2(2 \cdot 60)$ & 0.000338 \\
\hline $2-9$ & 2 & 0.020143 & & & \\
\hline $3-1$ & 2 & 0.020143 & $\mathrm{~N}(9)$ & $77(100)$ & 0.0130 \\
\hline $3-2$ & 1 & 0.010071 & & & \\
\hline $3-6$ & 2 & 0.020143 & & & \\
\hline $3-7$ & 1 & 0.010071 & & & \\
\hline $4-3$ & 2 & 0.020143 & & & \\
\hline $4-6$ & 2 & 0.020143 & & & \\
\hline $5-5$ & 1 & 0.010071 & & & \\
\hline $5-6$ & 2 & 0.020143 & & & \\
\hline $5-8$ & 1 & 0.010071 & & & \\
\hline $6-3$ & 2 & 0.020143 & & & \\
\hline $6-7$ & 3 & 0.030214 & & & \\
\hline $6-8$ & 1 & 0.019971 & & & \\
\hline $7-1$ & 2 & 0.020143 & & & \\
\hline $7-2$ & 4 & 0.040286 & & & \\
\hline $7-3$ & 16 & $0 \cdot 161143$ & & & \\
\hline $7-4$ & 1 & 0.010071 & & & \\
\hline $7-6$ & 11 & $0 \cdot 110786$ & & & \\
\hline $7-7$ & 6 & 0.060429 & & & \\
\hline $7-8$ & 1 & 0.010071 & & & \\
\hline $7-9$ & 1 & 0.010071 & & & \\
\hline $8-2$ & 1 & 0.010071 & & & \\
\hline $8-3$ & 2 & 0.020143 & & & \\
\hline $8-6$ & 1 & 0.010071 & & & \\
\hline $8-7$ & 1 & 0.010071 & & & \\
\hline $9-3$ & 2 & 0.020143 & & & \\
\hline $9-6$ & 1 & 0.010071 & & & \\
\hline $10-2$ & 2 & $0 \cdot 020143$ & & & \\
\hline $\mathrm{N}(37)$ & 98 & 0.9870 & & & \\
\hline
\end{tabular}

MICROSATELLITE DNA MARKERS AND DETECTION OF POLYMORPHISMS

Two markers used in this study, AFMa336zf9 and AFMb281wg9, were provided by Généthon Resource Center. These markers had earlier been shown to have the most significant linkage disequilibrium in the Finnish SD families. ${ }^{16}$ PCR based detection of microsatellite polymorphisms was carried out as previously described. ${ }^{715}$

\section{RISK CALCULATIONS}

Risk was computed using the MLINK program of the LINKAGE package. ${ }^{18}$ Haplotype frequencies were counted in the Finnish Salla families previously used for linkage analysis ${ }^{7}$ and were combined with those included in the risk calculations only. Altogether they consisted of 98 normal and 77 SD chromosomes. A three locus haplotype for each of the two marker loci and the Salla locus was constructed assuming a locus order of SD-AFMa336zf9AFMb281wg9 with zero recombination between the marker loci, as observed. ${ }^{16}$ The three locus haplotype frequencies were included in the analysis under the assumption that the observed linkage disequilibrium is valid and were adjusted to an estimated local SD population frequency of 0.013 (table 1). All the alleles observed (two for SD, 10 for AFMa336zf9, and nine for AFMb281wg9) were included in the haplotypes which then constituted a total of 180 possible haplotype combinations.

\section{Results}

The segregation of alleles in the 10 families at the two marker loci, AFMa336zf9 and AFMb281wg9, previously shown to be closely linked to the SD gene are shown in figs 1 and 2. Of the possible 180 haplotype combinations, 37 were observed in the normal chromosomes while only nine were present in the SD chromosomes (table 1). The results of the risk calculations with two marker haplotypes at different values of $\theta$ are shown in table 2 for the prenatal cases and in table 3 for the carrier identifications.

RISK CALCULATIONS IN PRENATAL CASES

In families 1 and 2 the risk calculation gave a probability level of $\geqslant 96.04 \%$ for an unaffected heterozygous fetus. In family 4 the first fetus (4) was predicted to be homozygous for the normal gene, since the probability of SD heterozygosity was $\leqslant 3.92 \%$, and the risk of being affected was below $0.04 \%$. The second fetus was predicted to be a healthy heterozygote with a probability of $\geqslant 96.08 \%$. In family 3 an affected fetus was predicted (homozygote for $1-3)$ with a probability level of $\geqslant 96 \cdot 04 \%$. All the results of the risk calculations are concordant with the prenatal studies performed during pregnancy by sialic acid assay.

CARRIER ANALYSIS

Carrier detection was based on the high predictive value of the risk haplotype $1-3$, which 
Table 2 Risks of carrier status (heterozygous 1/2) or affected (homozygous 2/2) phenotypes in four Finnish Salla disease families studied as prenatal diagnosis cases (fig 1)

\begin{tabular}{|c|c|c|c|c|c|c|c|c|}
\hline \multirow[b]{2}{*}{ Family } & \multirow[b]{2}{*}{ Subject } & \multirow[b]{2}{*}{ Salla genotype } & \multicolumn{5}{|c|}{ Risk (\%) at given $\theta$} & \multirow[b]{2}{*}{ Predicted phenotype } \\
\hline & & & 0.000 & 0.005 & 0.010 & 0.015 & 0.020 & \\
\hline 1 & 1 & $1 / 2$ & $97 \cdot 98$ & $97 \cdot 50$ & $97 \cdot 01$ & $96 \cdot 53$ & $96 \cdot 04$ & Healthy SD carrier \\
\hline 1 & 2 & $1 / 2$ & 0 & 0 & 0 & 0 & 0 & Healthy non-carrier \\
\hline \multirow[t]{2}{*}{1} & 3 & $1 / 2$ & $97 \cdot 98$ & $97 \cdot 00$ & 96.03 & 95.06 & $94 \cdot 10$ & Healthy SD carrier \\
\hline & & $2 / 2$ & 0 & 0 & 0 & 0 & 0 & \\
\hline \multirow[t]{2}{*}{2} & 4 & $1 / 2$ & 100 & $99 \cdot 00$ & $98 \cdot 01$ & 97.03 & $96 \cdot 07$ & Healthy SD carrier \\
\hline & & $2 / 2$ & 0 & $0 \cdot 50$ & 0.99 & $1 \cdot 48$ & 1.96 & \\
\hline \multirow[t]{2}{*}{3} & 4 & $1 / 2$ & 0 & $1 \cdot 00$ & 1.98 & $2 \cdot 96$ & 3.92 & \\
\hline & & $2 / 2$ & 100 & $99 \cdot 00$ & $98 \cdot 01$ & $97 \cdot 02$ & $96 \cdot 04$ & Affected with SD \\
\hline \multirow[t]{2}{*}{4} & 4 & $1 / 2$ & 0 & $1 \cdot 00$ & 1.98 & $2 \cdot 98$ & $3 \cdot 92$ & Healthy non-carrier \\
\hline & & $2 / 2$ & 0 & 0.00 & 0.01 & 0.02 & 0.04 & \\
\hline \multirow[t]{2}{*}{4} & 5 & $1 / 2$ & 100 & $99 \cdot 01$ & $98 \cdot 02$ & $97 \cdot 05$ & $96 \cdot 08$ & Healthy SD carrier \\
\hline & & $2 / 2$ & 0 & 0.50 & 0.99 & 1.48 & 1.96 & \\
\hline
\end{tabular}

Table 3 Risks of carrier status (heterozygous 1/2) or affected (homozygous 2/2) phenotypes in six Finnish Salla disease families studied as carrier diagnosis cases (fig 2)

\begin{tabular}{|c|c|c|c|c|c|c|c|c|}
\hline \multirow[b]{2}{*}{ Family } & \multirow[b]{2}{*}{ Subject } & \multirow[b]{2}{*}{ Salla genotype } & \multicolumn{5}{|c|}{ Risk (\%) at given $\theta$} & \multirow[b]{2}{*}{ Predicted phenotype } \\
\hline & & & 0.000 & 0.005 & 0.010 & 0.015 & 0.020 & \\
\hline 1 & 3 & $1 / 2$ & 0 & $1 \cdot 00$ & 1.98 & $2 \cdot 96$ & $3 \cdot 92$ & Non-carrier \\
\hline 1 & 5 & $1 / 2$ & 100 & $99 \cdot 50$ & $99 \cdot 00$ & $98 \cdot 50$ & 98.00 & Carrier \\
\hline 2 & 5 & $1 / 2$ & 0 & 0 & 0 & 0 & 0 & Non-carrier \\
\hline 2 & 6 & $1 / 2$ & 100 & $99 \cdot 50$ & $99 \cdot 00$ & $98 \cdot 50$ & $98 \cdot 88$ & Carrier \\
\hline 2 & 7 & $\begin{array}{l}1 / 2 \\
2 / 2\end{array}$ & $\begin{array}{l}0 \\
0\end{array}$ & $\begin{array}{l}0 \cdot 50 \\
0\end{array}$ & $\begin{array}{l}0.99 \\
0\end{array}$ & $\begin{array}{l}1 \cdot 48 \\
0\end{array}$ & $\begin{array}{l}1 \cdot 96 \\
0\end{array}$ & Non-carrier \\
\hline 2 & 8 & $1 / 2$ & 100 & $99 \cdot 50$ & 99.00 & $98 \cdot 50$ & $98 \cdot 00$ & Carrier \\
\hline 2 & 9 & $1 / 2$ & 0 & 0.50 & 1.00 & $1 \cdot 50$ & $2 \cdot 00$ & Non-carrier \\
\hline 3 & 15 & $1 / 2$ & 100 & $99 \cdot 50$ & 99.00 & $98 \cdot 50$ & $98 \cdot 00$ & Carrier \\
\hline 3 & 18 & $1 / 2$ & 0 & 0.50 & 1.00 & 1.50 & $2 \cdot 00$ & Non-carrier \\
\hline 3 & 19 & $1 / 2$ & 0 & 0 & 0 & 0 & 0 & Non-carrier \\
\hline 3 & 20 & $1 / 2$ & 100 & $99 \cdot 50$ & $99 \cdot 00$ & $98 \cdot 50$ & $98 \cdot 00$ & Carrier \\
\hline 3 & 21 & $1 / 2$ & 0 & 0.50 & 0.99 & $1 \cdot 48$ & 1.96 & Non-carrier \\
\hline 3 & 22 & $1 / 2$ & 0 & $0 \cdot 50$ & 0.99 & $1 \cdot 48$ & 1.96 & Non-carrier \\
\hline 4 & 1 & $1 / 2$ & 100 & $99 \cdot 50$ & $99 \cdot 00$ & $98 \cdot 50$ & $98 \cdot 00$ & Carrier \\
\hline 4 & 2 & $1 / 2$ & 0 & $1 \cdot 00$ & 1.98 & $2 \cdot 96$ & 3.92 & Non-carrier \\
\hline 4 & 5 & $1 / 2$ & 100 & $99 \cdot 50$ & $99 \cdot 00$ & $98 \cdot 50$ & $98 \cdot 00$ & Carrier \\
\hline 5 & 3 & $1 / 2$ & 0 & $1 \cdot 00$ & 1.98 & $2 \cdot 96$ & 3.92 & Non-carrier \\
\hline 5 & 4 & $1 / 2$ & 0 & 0 & 0 & 0 & 0 & Non-carrier \\
\hline 5 & 5 & $1 / 2$ & 100 & $99 \cdot 50$ & $99 \cdot 00$ & $98 \cdot 50$ & $98 \cdot 00$ & Carrier \\
\hline 5 & 6 & $1 / 2$ & 100 & 99.00 & $98 \cdot 00$ & $97 \cdot 00$ & $96 \cdot 00$ & Carrier \\
\hline 5 & 7 & $1 / 2$ & 0 & 0.50 & $1 \cdot 00$ & $1 \cdot 50$ & $2 \cdot 00$ & Non-carrier \\
\hline 6 & 4 & $1 / 2$ & 100 & 99.01 & $98 \cdot 02$ & $97 \cdot 05$ & $96 \cdot 08$ & Carrier \\
\hline & & $2 / 2$ & 0 & 0.50 & 0.99 & $1 \cdot 48$ & 1.96 & \\
\hline
\end{tabular}

has so far been found only on one normal chromosome in the Finnish population. In at risk families, subjects with high risk values $\geqslant 96.08 \%$ were considered to be carriers of the SD gene and those with risk values $\leqslant 3.92 \%$ were identified as non-carriers. In family 5 a new mutation at the marker locus AFMb281wg9 was detected either in subject 3 or 9 , in whom either allele 4 or 5 must have been mutated. However, this had no effect on the risk calculations. Subjects without a family history of SD (spouses of family members) had carrier risk levels of $0 \%$. The use of an SD gene frequency of 0.006 had no effect on the risk calculation. With lower $\theta$ values of 0.005 $0 \cdot 000$, the corresponding risk estimations were $\geqslant 99.01 \%$ for carriers and $\leqslant 1 \%$ for non-carriers.

The risk was also calculated for four newborn infants, at the request of the parents, to exclude the disease. In family 2 the baby's (7) haplotype yielded a $0 \%$ risk of being affected and the carrier probability was $\leqslant 1.96 \%$, depending on the chosen $\theta$. The free sialic acid in the baby's urine was within the normal range. The child is now 6 months old and has shown no evidence of developmental delay or any other signs of SD. In family 3 the DNA of the twin girls (21) and (22) was extracted from the placentas after birth, and their haplotypes gave risk levels $\leqslant 1.96 \%$ of being a carrier. No urine tests have been performed. The girls are now 14 months of age and have developed normally. In family 6 the baby's (4) urine was examined shortly after birth and normal levels of free sialic acid were detected, suggesting a healthy child. DNA analysis predicted him to be an SD heterozygote, with a probability of $\geqslant 96.08 \%$, compared to the risk of being affected of $\leqslant 1.96 \%$. The boy is now 16 months of age and has reached the normal milestones of development.

In family 7 the healthy mother was found to be homozygous for the 1-3 haplotype, representing so far the only 1-3 haplotype in a normal, non-SD chromosome. At the time of the DNA analysis the diagnosis of her son, the first child of unrelated parents, was still equivocal but SD was strongly suspected on the basis of his clinical findings. His subsequent clinical course, repeated free sialic acid assays of urine, and findings of demyelination of the central nervous system on MRI has confirmed the diagnosis of SD. The results of the haplotype analysis with 1-3 homozygosity are thus fully consistent with the phenotypic findings. However, if an unknown phenotype was assumed for the baby (as in a prenatal situation), his risk of being an SD carrier would be $\geqslant 49.98 \%$ or $\geqslant 41.08 \%$ and the risk of being affected $\geqslant 16.87 \%$ or $\geqslant 8.01 \%$, with an SD gene frequency of 0.013 or 0.006 , respectively. 


\section{Discussion}

Previous experience with the present families indicates that the determination of the level of free sialic acid (NANA) from uncultured chorionic villus samples is an accurate and reliable method for prenatal diagnosis of SD (M Renlund, personal communication). One of the five fetuses in the present study material had been found to be affected on the basis of raised free sialic acid levels in the uncultured chorionic villus sample. The pregnancy was terminated and the diagnosis was confirmed by EM and free sialic acid assays of fetal tissues. Prenatal diagnosis of SD had been attempted previously with cultured amniocytes by free sialic acid and free/total sialic acid ratio determination. ${ }^{11}$ However, cultured amniotic fluid cells are not fully appropriate because the increase in NANA in SD is only moderate and the effect of the culture conditions is unknown. In ISSD, the other phenotypic manifestation of lysosomal sialic acid storage, the more pronounced rise of intracellular free sialic acid has provided an even more straightforward approach for prenatal diagnosis. ${ }^{12-14}$

The present study shows that as a result of the assignment of the SD locus to $6 \mathrm{q}$, linkage analysis is an alternative method for prenatal diagnosis. The strong linkage disequilibrium observed in the Finnish SD families with microsatellite markers AFMa336zf9 and AFMb281wg9 ${ }^{16}$ substantially adds to the power of linkage analysis. No recombinations have yet been detected between these two markers and the SD locus. The risk haplotype 1-3 has been detected in $91 \%$ of parental Finnish SD chromosomes but only once in a normal chromosome, the mother of family 7 in the present study. The haplotype frequencies, together with the segregation of the disease in the SD families, determine the risk which is largely independent of the gene frequency given strong linkage disequilibrium. The present case is a good example of this. The correctness of the risk calculation depends on the accuracy of the estimation of haplotype frequency, primarily of that of the high association haplotype in normal chromosomes.

The retrospective risk calculations correctly predicted the phenotype of the fetus in all the families. In the other recessive diseases enriched in the Finnish population, prenatal diagnosis using linkage disequilibrium has previously successfully been used in diastrophic dyplasia, ${ }^{19}$ infantile ceroid lipofuscinosis (CLN1), ${ }^{20}$ and cartilage-hair hypoplasia. ${ }^{21}$ In these diseases the degree of allelic associations and the level of risk probabilities have been approximately within the same range as in SD. In non-Finnish families conventional linkage analysis can now be used for prenatal diagnosis in both SD and ISSD since no locus heterogeneity was detected in our previous linkage studies of different phenotypes of free sialic acid storage diseases. ${ }^{16}$

A possible source of error is mutation of microsatellites, as could be seen in family 5 in our carrier studies. So far this is the only mutation event detected with markers AFMa336zf9 and AFMb281wg9 from about 500 chromo- somes analysed. The estimated average mutation rate of microsatellites, $10^{-4}$, is still relatively $l o w^{22}$ and data from various studies suggest that microsatellites amplify accurately enough to be suitable for diagnostic studies. Our calculations were also carried out assuming a mutation frequency of $10^{-3}$ for the marker AFMb281wg9 but this had no effect on the risks. A possibility remains that the only detected $1-3$ non-SD haplotype in family 7 results from a new mutation either in AFMa336zf9 or AFMb281wg9. However, no direct evidence for this was detected when the extended haplotypes of the parents of the 1-3 homozygous mother were analysed.

In the past, carrier identification in SD was not possible. For subjects with no previous history of SD and not originating from the high risk area of Salla, a risk estimation was calculated using both gene frequencies, 0.006 and $0 \cdot 013$. The gene frequency estimation of 0.006 was based on the annual incidence of about two SD cases per 60000 births. $^{7}$ For those with no family history of SD but originating from the high risk area, a disease gene frequency of 0.013 was used. This was based on the carrier frequency estimation of 1:40 in this area. ${ }^{23}$ However, the different gene frequencies had no significant effect on the calculated risks in these cases.

As mentioned above, the different estimates of the SD gene frequency have little effect on the risk of probabilities in the SD families because their risks are based on segregation and linkage. However, in a case of a person from the general Finnish population, the risk is based on the frequencies of the SD gene and the 1-3 risk haplotype. We can now estimate that the probability for any Finnish person carrying the 1-3 risk haplotype of being an SD carrier is approximately $33 \%$ or $51 \%$ depending on the SD gene frequency of 0.006 or 0.013 . The corresponding figures for a 1-3 homozygote would be $\sim 44 \%$ or $50 \%$ of being a carrier and $\sim 10 \%$ or $25 \%$ of being affected. The probability for a subject without the 1-3 haplotype of being an SD carrier can be estimated from the haplotype frequencies in normal and SD chromosomes (table 1). The risk associated with all other haplotypes present in both normal and SD chromosomes is very low, ranging from 0.1 to $1.5 \%$. Accordingly, the rare haplotypes, such as $1-8$ which has so far been found in SD chromosomes only, would indicate a high risk for heterozygosity but this is probably because of the limited number of chromosomes studied so far. These estimates are substantially more accurate than the predictions based on the gene frequencies alone. Haplotype analysis can therefore be used in clinical practice to predict the risk of SD. A couple who both lacked the 1-3 risk haplotype would have a low risk of bearing an affected child whereas a couple with both parents having the high risk haplotype would run a considerable risk of having a child with SD. The haplotype analysis will, however, mostly benefit the family members of the SD patients who wish to know their carrier status. As shown by 
the present results, haplotype analysis can give reliable predictions in most cases.

Cloning of the SD gene and identification of the mutation causing the disease will eventually replace the haplotype analysis in carrier detection and in prenatal diagnosis, at least in the Finnish population. The observed linkage disequilibrium strongly suggests that there is only one major mutation causing the disease in this founder population, as is the case with aspartylglucosaminuria and very likely also with the other genetic diseases enriched in the Finnish population. ${ }^{24}$ The presence of only one or a few mutations would facilitate diagnostic studies and even provide the possibility for carrier screening in the general population.

The cooperation and positive attitude of all the SD families is greatly appreciated. We thank Pirkko Jalava for her assistance in the laboratory. This study was financially supported by the Maud Kuistila Foundation, Finland and the Academy of Finland.

1 Renlund M, Tietze F, Gahl WA. Defective sialic acid egress from isolated fibroblast lysosomes of patients with Salla from isolated fibroblast lysosomes
disease. Science 1986;323:759-62.

2 Mancini GMS, Verheijen FW, Beerens CEMT, Renlund $M$, Aula P. Characterization of a proton-driven carrier for sialic acid in the lysosomal membrane: evidence for a group-specific transport system for acidic monoaccharides. F Biol Chem 1989;264:15247-54.

3 Mancini GMS, Beerens CEMT, Aula P, Verheijen FW Sialic acid storage diseases. A multiple lysosomal transport defect for acidic monosaccharides. F Clin Invest 1991;87 1329-35.

4 Renlund M, Aula P, Raivio KO, et al. Salla disease: new lysosomal storage disorder of disturbed sialic acid metabolism. Neurology 1983;33:57-66.

5 Aula P, Autio S, Raivio KO, et al. "Salla disease": a new lysosomal storage disorder. Arch Neurol 1979;36:88-94.

6 Renlund M. Clinical and laboratory diagnosis of Salla disease in infancy and childhood. 7 Pediatr 1984;104:232-6.

7 Schleutker J, Laine AP, Haataja L, et al. Linkage disequilibrium utilized to establish a refined genetic position of the Salla disease locus on 6q14-q15. Genomics 1995: 27:286-92.

8 Echenne B, Vidal M, Maire I, Michalski JC, Baldet P, Astruc J. Salla disease in one non-Finnish patient. Eur $\mathcal{F}$ Pediatr 1986;146:320-2.
9 De Kremer RD, de Boldini CD, de Capra AP, Hliba E. Enfermedad de Salla (sialuria, tipo Finlandese); nueva variante clinica en un primer reconocimiento Argentino. Medicina 1990;50:107-16.

10 Tondeur M, Libert J, Vamos E, Van Hoof F, Thomas GH, Strecker G. Infantile form of sialic acid storage disorder: clinical, ultrastructural, and biochemical studies in two siblings. Eur 7 Pediatr 1982;139:142-7.

11 Renlund M, Aula P. Prenatal detection of Salla disease based upon increased free sialic acid in amniocytes. $A m$ 7 Med Genet 1987;28:377-84.

12 Vamos E, Libert J, Elkhazen N, et al. Prenatal diagnosis and confirmation of infantile sialic acid storage disease. Prenat Diagn 1986;6:437-46.

13 Clements PR, Taylor JA, Hopwood JJ. Biochemical characterization of patients and prenatal diagnosis of sialic acid storage disease for three families. F Inher Metab Dis 1988;11:30-44.

14 Lake BD, Young EP, Nicolaides K. Prenatal diagnosis of infantile sialic acid storage disease in a twin pregnancy. $\mathcal{F}$ Inher Metab Dis 1989;12:152-6.

15 Haataja L, Schleutker J, Laine AP, et al. The genetic locus for free sialic acid storage diseases maps to the long arm for free sialic acid storage diseases maps to the long arm

16 Schleutker J, Leppänen P, Månsson JE, et al. Lysosomal free sialic acid storage disorders with different phenotypic presentations, ISSD and Salla disease, represent allelic disorders on 6q14-15. Am f Hum Geneti (in press).

17 Dubeau L, Chandler LA, Gralow JR, Nichols PW, Jones PA. Southern blot analysis of DNA extracted from formalin-fixed pathology specimens. Cancer Res 1986;46:2964 9.

18 Lathrop GM, Lalouel JM, Julier C, Ott J. Strategies for multilocus linkage analysis in humans. Proc Natl Acad $\mathrm{Sci}$ USA 1984;81:3443-6.

19 Hästbacka J, Salonen R, Laurila P, de la Chapelle A, Kaitila I. Prenatal diagnosis of diastrophic dysplasia with polyI. Prenatal diagnosis of diastrophic dysplasia with po
morphic DNA markers. 7 Med Genet 1993;30:265-8.

20 Vesa J, Hellsten E, Mäkelä TP, et al. A single PCR marker in strong allelic association with the infantile form of neuronal ceroid lipofuscinosis facilitates reliable prenatal diagnostics and disease carrier identification. Eur $f$ Hum Genet 1993;1:125-32.

21 Sulisalo T, Sillence D, Wilson $M$, Ryynänen $M$, Kaitila I. Early prenatal diagnosis of cartilage-hair hypoplasia CHH) with polymorphic DNA markers. Prenat Diagn 1995; 15:135-40.

22 Jorde LB, Watkins WS, Viskochil D, O'Connell P, Ward K. Linkage disequilibrium in the neurofibromatosis I (NFI) region: implications for gene mapping. Am f Hum Genet region: implications

23 Aula P, Renlund M, Raivio K. Screening of inherited oligosaccharidurias among mentally retarded patients in Northern Finland. $\mathcal{F}$ Ment Defic Res 1986;30:365-8.

24 De la Chapelle A. Disease gene mapping in isolated human populations: the example of Finland. $\mathcal{F}$ Med Genet 1993; 30:857-65. 